\title{
Research on the Emergence Modeling of Equipment System
}

\author{
Xin-Hua He ${ }^{1}$, Wei-Chao Zhang ${ }^{2}$, Qiang Qu ${ }^{3}$, Wan-Lin Lu ${ }^{4}$ \\ ${ }^{1}$ Department of Information Engineering, Academy of Armored Force Engineering, Beijing, China \\ ${ }^{2}$ Department of Information Engineering, Academy of Armored Force Engineering, Beijing, China \\ ${ }^{3}$ Department of Information Engineering, Academy of Armored Force Engineering, Beijing, China \\ ${ }^{4}$ Troop No.66133 of PLA, Beijing, China \\ 1hxh717495@sina.com, ${ }^{2} 342722719 @ q q . c o m,{ }^{3} 535565543 @ q q . c o m,{ }^{4} 786164744 @ q q . c o m$
}

\begin{abstract}
Under the conditions of information, the network-centric system and the confrontation in the system has developed into a major combat style. But the traditional line of sexual assessment method is difficult to accurately assess the information equipment system combat capability. Therefore, this paper studies the effective evaluation method of the operational capability of the information equipment system from the perspective of emerge. Based on the simulation modeling and evaluation method, building the capability model of the weapon equipment system to evaluate the operational capability of the information weapon weaponry equipment. Through the example analysis, the validity of the simulation model and the practicability of the evaluation system is analyzed by analyzing the examples.
\end{abstract}

\section{Introduction}

Weapons and equipment system is the basis of warconfrontation system, and it is also the basis of war system confrontation, its ability depends on the strength of the system's inherent combat capability, it is not only the sum of equipment and system combat capability, or the composition of the system structure and the composition of the interaction between the system [1], thus the emergence is the overall combat capability. However, the existing evaluation methods have many shortcomings in the evaluation of the overall emergence capability of the combat capability of the weapon equipment system. Therefore, this paper puts forward the main unit model of the combat capability of the equipment system by studying the theory of the combat capability of the weapon equipment system, by analyzing the method of multi-data association, the relationship between the principal units is judged, and building the weapon equipment system combat capability appearance model, evaluating the combat capability of it.

\section{Information Weapon Equipment System Operational Ability}

\subsection{Brief Introduction to the Combat Ability of the Information Weapon Equipment System}

Information-based weapons and equipment system combat capability is generated through the component system [2]. System within the various components of the system is a certain composition between the structure of interaction, resulting in the emergence of the effect form the whole. System combat capability is a new combat capability that emerges in this process, and these new combat capabilities are not equal to the linear sum of the original component system combat capability.

The information technology system is formed by the development of information network technology, its system combat capability generation has the characteristics of complexity, factor independence, factor synergy, overall emergence and so on.

\subsection{Research Status of Combat Capability Evaluation of Weapon Equipment System}

Since the birth of weapons and equipment, the calculation and evaluation of its combat capability has always been the command staff at all levels of attention. Many experts and scholars at home and abroad have made many pioneering researches on the evaluation of the combat capability of the weaponry and equipment system. In summary, the main methods of system combat capability assessment are divided into four categories: expert evaluation method, multi-index comprehensive method, test statistical method, modeling and simulation class method [3].

Expert evaluation method has the advantage of judging intuitive, less binding, easy to understand. The disadvantage is subjective and the evaluation results are not stable. The advantage of multi-index synthesis method is that the calculation model is clear, more scientific and reasonable, but the calculation model has few factors to consider, the lack of expression of the system. The advantage of the statistical method is that the evaluation 
results are more accurate, but it requires a large amount of statistical data to be analyzed. The advantage of the modeling and simulation method is that it takes into account the structure and interaction of the combat capability of the system, but the reliability of the simulation model is difficult to test and the computational complexity is high.

\section{The Explanation of the Relevant Concept Of Emancipation}

\subsection{The Concept of the System Combat Capability Emerged}

Emergence is a transition from low to high level [4], it is an overall emergence of the composition of the composition of the system in accordance with the structure of the interaction, and each complement, the effect is mutual restraint.

The emergence of combat capability means that the underlying combat capability is integrated and combined with a certain relationship form a new operational capability to meet the needs of operational using. It can get a higher level of combat capability through the ability of emerge, and it can also get the original capacity which not have some of the characteristics, these features will be more intuitive to show the strength of combat capability[5].

Therefore, we can give the definition from the combat capability of the weaponry and equipment system. Through the combination of the certain organizational structure and the certain level of the characteristics, the integration and combination of the combat capability of the weapon equipment system forms a new and value-added overall combat capability, so as to meet the needs of weapons and equipment system against combat.

\subsection{The Mechanism of System Combat Capability Emerges}

\subsubsection{The Reason of System Combat Capability Emerges}

The emergence of the three elements are a large number of the main body, the interaction between the main body, a group of simple rules of the conduct. The reason for the emergence of system combat capability is that in the equipment system with all kinds of equipment in the information system supports the battlefield situation sharing, coordination, interactive feedback of the system capacity.

\subsubsection{The Manifestation of System Combat Capability Emerges}

The operational elements within the equipment system are driven because of a common goal, dynamic, adaptive and interdependent to achieve the synergies, and the collaboration can improve the combat capability in a variety of ways. Combat elements of the coordination can be collaborative feedback. So System combat capability gets emerged in the form of performance for the combat capability of collaborative feedback, the formation is a new overall combat capability [6].

1) The Feature of System Combat Capability Emerges

Information ability is the basis of system combat capability. It is a variety of elements of centralized control, unified coordination, composed of a certain spatial structure to produce. So the interaction of a large number of elements is the reason of the emergence of system combat capability. and the performance of the system combat capability is the size and state of a factor's ability to have different degrees of feedback on the size and state of the associated factor ability.

\subsection{Based on the Emerging System of Combat Capability Assessment Framework}

The emergence of system combat capability is the ability of the system to a certain structural relationship. Therefore, the core of the model is to establish an effective system capacity model, unit structure model, and unit action model.

The system capacity model can reflect the characteristics of the system capacity and the process of system capacity generation. The unit structure model can accurately infer the structural relationship between the system capacity constituent elements, which indicates the occurrence of structural phenomena and the relationship between the emergence of the phenomenon. The model of unit action reflects the interaction between units, which makes the system capacity have different results.

\section{Constructing The Main Unit Model Of The Bottom Combat Capability}

\subsection{Method of Building a Model}

The main unit of the combat capability is the microscopic unit of the system combat capability, which is the foundation of the system to build the combat capability.

The method of constructing the main unit model of the bottom combat capability refers to the basic steps of constructing the required subject model and the core of the research problem. This paper refers to the CGF model, combined with the characteristics of the field of weapons and equipment system, and using a set of combat capability for its own characteristics of the main combat capability modeling method. The basic process is as follows:

Step 1: Define the domain and characteristics of the competency unit.

The ability of the main unit is an autonomous individual, characterizing a weapon equipment system combat capability behavior characteristic. These principal units generate system combat capability through cooperative relationships.

Step 2: Constructing the mapping relationship between the weapon system and capacity main unit.

From the composition of weapons and equipment 
system to start, analyzing the system at all levels deeply, establish the weapons and equipment system structure to the combat capability of the main unit mapping.

Step 3: Constructing the hierarchy structure of the main unit of combat capability of weapon equipment system.

Capability unit hierarchy refers to the specific form of spatial arrangement and combination of the main body units within the system. Hierarchical combing system combat capability of the main unit, form a certain level structure.

Step 4: Judge capability unit associated structure.

The complex relationship between the elements of the equipment system is manifested as the complex relationship between the elements of the capacity. Analyze the relationship further between the main unit of the combat capability of the system, the network-like capacity of the main unit spatial structure.

Step 5: Inspection and evaluation.

To complete the establishment of a network-like system combat capability of the main unit spatial structure, inspection and correction of the main capacity of the main unit and the composition of the spatial structure of the actual impact of the actual using of weapons and equipment characteristics referring to the force drill data.

\subsection{Determine the Relationship between the Main Unit of the Operational Capability of the System}

The emergence of the system combat capability is the combat capability of the main unit with a certain composition and interaction of the resulting, so this article determines the weapons and equipment combat capability of the main unit relationship based on the SEM.

Step 1: Collect the sample size, the number of 10 times the unknown parameters

Step 2: Draw the RAM map, get the system combat capability of the main unit of the relationship by its topology. Then determine the estimator of the parameters in the model according to the $t$ rule.

Step 3: By referring to the method of maximum likelihood method to estimate the association relation model, and then through the likelihood function $F_{M L}=\ln |\Sigma(\theta)|+\operatorname{tr}\left[\Sigma^{-1}(\theta) S\right]$ to iterate a set of parameters $\theta$ which makes $F_{M L}$ to reach the minimum value.

Step 4: Evaluate the model that has been obtained.

Step 5: Modify the association relationship model, modify the part that is not realistic.

\section{Research on the Emergence Model Of Combat Ability of Weapon Equipment System}

\subsection{The Relationship Model of System Combat Capability}

5.2.1 Correlation and Dynamics Model of System Combat
Capability

Based on Kuramoto's coupled oscillator synchronization model to establish the system combat capability of the main unit combat collaborative dynamics model ${ }^{[8]}$ :

$$
\frac{d \theta_{i}}{d t}=\omega_{i}+c_{i j} \sum_{j=1}^{N} a_{i j} \sin \left(\theta_{i}-\theta_{j}\right), i=1,2, \cdots
$$

The state of the combat capability subject unit $i$ changing with time can be described by the phase parameter $\theta_{i}(t)$. Its operational command cycle is $\mathrm{T}_{i}=2 \pi / \omega_{i}, A=\left(a_{i j}\right) \in R^{N \times N}$ is its adjacency matrix, if there is an association between the competency unit $i$ and $j$, then $a_{i j}=a_{j i}=1$; else $a_{i j}=a_{j i}=0, c_{i j}$ is the correlation strength between the capability main unit $i$ and $j$.

\subsubsection{System Combat Capability Correlation Relation} Interaction $\mathrm{Model}$

In the combat capability of the main unit weapons and equipment system shows that a combat capability of the main unit of another one of the gain or inhibition to the interaction relationship [9].According to the combat capability of the main unit of the feedback to characterize the impact of coordination on the system combat capability. When the combat capability $C_{1}$ and $C_{2}$ mutual get influence, the combat capability gets changed, the expression is as follows:

$C_{2}^{\prime}=\left\{\begin{array}{l}C_{2}+\Delta t_{1}, \rho \geq \sigma_{2} \\ C_{2}, \sigma_{1} \leq \rho<\sigma_{2} \\ C_{2}-\Delta t_{2}, \rho<\sigma_{1}\end{array}\right.$

Where $\Delta t$ is the relationship between the feedback effect, $\rho$ is the degree of correlation between the ability of the main unit, Define the degree of association of the main unit of combat capability is $\rho_{i j}(t)=\left\langle\cos \left[\theta_{j}(t)-\theta_{i}(t)\right]\right\rangle,-1 \leq \rho_{i j} \leq 1,\left[\sigma_{1}, \sigma_{2}\right]$ is

the degree of association. When the degree of association exceeds the threshold, the combat capability $C_{2}$ has an enhanced effect; otherwise, there is a decay effect, the degree of coordination in a range of combat capability $C_{2}$ remain unchanged.

\subsection{A New Model that Similar to the Cellular Automata Model}

On the basis of cellular automata, combined with the demand for the emergence of weapons and equipment system combat capability, make the finite discrete state 
setting extend to the continuous space, and an improved cellular automata model is established.

Define an improved continuous space cellular automata model, it can be represented by a five-tuple: $A^{+}=\left(L, S_{d}, N^{+}, \theta, f\right)$, where $L$ represents the set of cells in the spatial structure; $S_{d}$ represents the continuous space of the cell state values. In the cellular automata, the cell state value is infinite continuous space, where $d$ is the state space dimension; $N^{+}$is the neighbor of the cell; $\theta$ is the phase of the cell; $f$ is the state evolution function of the cell, cell's phase changed rules and cellular state transition rules.

Compared with the traditional cellular automata, the continuous space cellular automata redefine the spatial range of the cell neighbors, and extends to the infinite continuous space from the original mesh space, making it more suitable for the non-discrete system model establishment.

\subsection{Emergence Model of Cellular Automata in Continuous Space}

\subsubsection{Cell's Unit}

Definition: In the associated cell automata model which in the system combat capability emerges, the main unit of system combat capability is the system combat capability of the cells, $C_{i}$ is the $i$ th cell, and the cell space composed of all the cells is $L$, so $C_{i} \in L$,

$i=1,2, \cdots$

\subsubsection{Cell's State}

In the association automata model which is defined in this paper, the unit of the system combat capability is the individual of the cell, and the combat capability value is the state value of the cell. The cell state value is the continuous value in the one-dimensional space. Therefore, the state value of the main unit of the system combat capability is the cell state which is essentially the value of the combat capability, and $S$ is the continuous space of all the cell states. In this paper, $S$ takes the interval $[0,10]$.

\subsubsection{Cell's Neighbors}

The neighbors of the capability cells are associated with the cells' phase changed and the state transition has an impact on all cell sets, that are the combat capability of the main unit of the combat capability.

\subsubsection{Evolution Rules of Cellular States}

The evolution of the associated cell system requires two conditions: the initial state of the cell and the evolution rules. The following show the evolution of the state of the cells:

1. Changing rules of the cell's phase

$\frac{d \theta_{i}}{d t}=\omega_{i}+c_{i j} \sum_{j=1}^{N} \sin \left(\theta_{i}-\theta_{j}\right), i=1,2, \cdots$

In the formula, the phase value of cell $i$ is $\theta_{i}$, which is determined by the influence of its own operational command cycle $\mathrm{T}_{i}=2 \pi / \omega_{i}$, and the influence of the relevant neighbor $N . c_{i j}$ is the correlation intensity between cell $i$ and $j$.

2.The transferring rules of cellular states

$s_{t+1}^{i}=s_{0}^{i}+\sum_{j=1}^{N} \lambda_{i j} \rho_{i j}$

In the formula, the state of cell $i$ at time $t+1$ is determined by the correlation between its initial state $s_{0}^{i}$ and its neighbor $N . \quad \rho$ is the degree of correlation between cell units, where $\rho_{i j}(t)=\left\langle\cos \left[\theta_{j}(t)-\theta_{i}(t)\right]\right\rangle$ and $-1 \leq \rho_{i j} \leq 1, \lambda_{i j}$ is the correlation coefficient.

\section{1) Evolution Rules of Cellular Systems}

Continuous space cellular-automata simulation weapon equipment system combat capability according to the relationship between its neighbors, the specific process is as follows:

Step 1: Construct the cellular automata model, configures the spatial environment of the cell, set the neighbor of the cell and specifies the evolution rule of the cell's state.

Step 2: Initialize the initial state value of the cell's body.

Step 3: Define the end of the system evolution, and define the critical value $\sigma$.

Step 4: Set the initial time for the evolution of the system $T_{0}$.

Step 5: Calculate the state-value of the cell's state at a certain time according to the state evolution rule.

Step 6:Calculate the amount of change $\Delta$ in the statevalue of a cell.

Step 7: Determine whether the system termination condition is less than $\sigma$, then jump to Step8, otherwise Step5.

Step 8: Stop the simulation of system, and output the combat capability cells' state-values.

\section{The Effectiveness Validation Of The Emerging Model}

\subsection{Emergence Model of Cellular Automata in Continuo}

Taking the weapon system of a military digitizer as an 
example, through the decomposition of combat capability, the weapon system of the army digitizer is composed of the investigation intelligence system, command and control system, comprehensive security system, fire power system, tactical communication system, electronic warfare system. According to the steps of modeling the combat capability unit of weapon equipment system, construct the main unit model of combat, and the data processing software of SEM is used to process the data, then judge the relationship between the main elements of weapon equipment combat capability.

\subsection{Simulation of the Model}

6.2.1 Experiment 1: Compare with the Traditional Modeling Methods

Aiming at the change of the combat capability of the weapon system after the information transformation, and then evaluate the combat capability of the system by using the correlation model and the traditional non - emergence model, and then compare the evaluation results of the two methods. The following methods are used to assess the combat capability of the Army digitizer's weaponry system:

(1) Assess the combat capability of the information weapons and equipment system through the relationship between the emerging model;

(2) Assess the combat capability of the information weapons and equipment system through the traditional non - emerging model;

The concrete results are shown in the figure 1 and figure 2 .

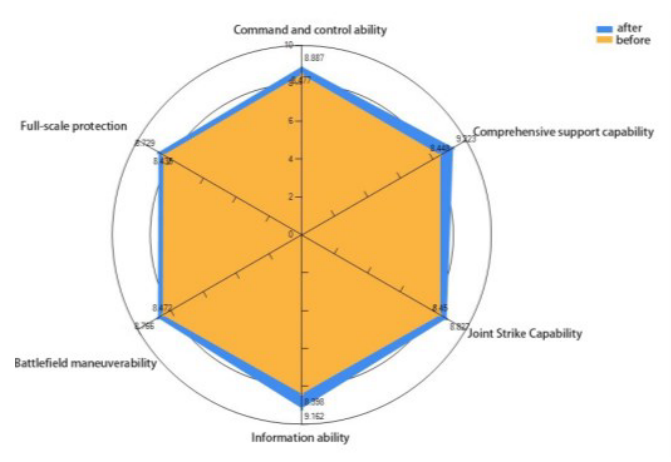

Figure 1. Combat capability contrast picture

Figure 1 shows the contrast of the system combat capability before and after the emergence. Figure 2 shows the compare of the ability of the weapons and equipment system before and after the emergence.

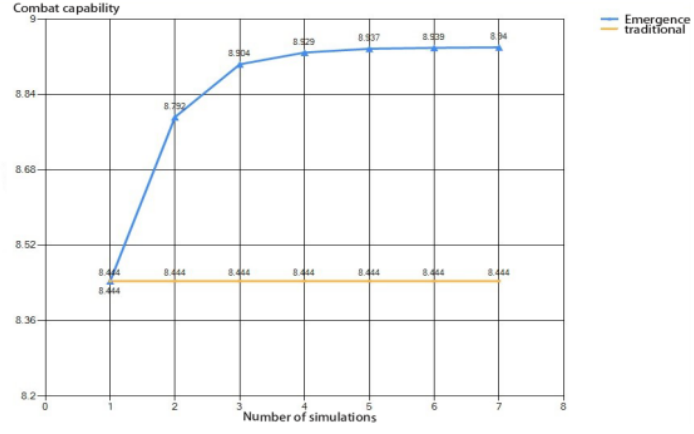

Figure 2. Combat capability contrast picture

It can be seen from Fig. 1 and 2 that the combat capability of the association model is higher than the traditional non-emerging model for the information weapon system. Therefore, information technology is helpful to improve the degree of combat capability, and the capabilities affect each other. So the model is used to evaluate the combat capability value higher than the traditional non-emerging model.

After the demand-demonstration and simulationexercises of the weapons and equipment, the division of the weapons and equipment system combat capability is assessed as 9.461 by the experts. Using the traditional nonemergence model simulation, due to the neglect of the relationship between the system capacity and the lack of combat capability associated with the emergence of the assessment, the result is 8.479 , the result of the association model presented in this paper is 9.201 , which is more practical.

6.2.2 Experiment 2: Analyze the Sensitivity of the Key Combat Capability

Considering the characteristics of the information system combat capability, this paper analyzes the sensitivity of the key operational capability in the system, that is the impact of key operational capability on the overall combat capability of the system, which reflects the validity of the model, the following experiments were conducted:

(1) for the digital transformation of the weapons and equipment system, analysis the sensitivity of the command and control ability;

(2) for the digital transformation of the weapons and equipment system, analysis the sensitivity of the information ability.

The concrete results are shown in the figure 3.

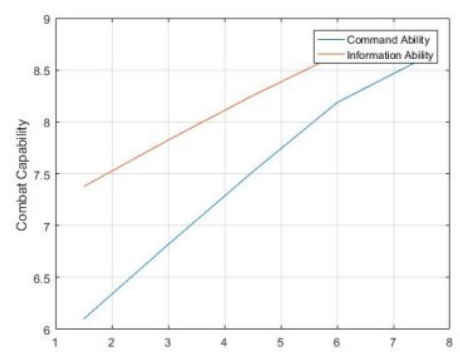

Figure 3. Analysis of sensitivity of key operational capability 
From the experimental results of the two experiments, we can see that: to the overall combat capability of the system, is a non-linear relationship. Through the experimental data, we can find that the model of the correlation relationship in this paper considers the interaction between the combat capability of the system and it is a non-linear evaluation method. Therefore, the model of the correlation relationship proposed can reflect the characteristics truly of the combat capability of the weapon system and the real situation of the combat capability.

\section{Conclusion}

This paper takes the evaluation model of the combat capability of weapon equipment system as a research object, and establishes a model of combat capability based on an improved cellular automaton, and deeply studies the simulation of the operational capability of weapon equipment system, then builds the weapon unit system combat capability main unit model.

However, the model is a very complex problem, the emerging model of the operational capability of the system is only a meaningful exploration, there are many shortcomings, and is needed to continue to study the mechanism and the form of expression. The weapons and equipment system of information will consider more and more external factors and forms of expression, so as to further refine and understand the emerging phenomenon.

\section{References}

[10]-of-Systems[M], 2011: 1-16.
[1] Charles Domercant, Jean, Mavris, Dimitri N. Measuring the architectural complexity of military Systems-of-Systems[C]//Proceedings of IEEE Conference, 2011.

[2] Li Guoyi, Meng Fanliang, Dong Zhengbao, et al. Modeling for military system by weighted complex networks[C]//Proceedings of 2010 8th IEEE International Conference on Control and Automation(ICCA), 2010.

[3] Stenner Jr, Charles E. Command making progress toward achieving full operational capability[J]. Citizen Airman, 2011, 63(3): 2-2.

[4] John Holland. Emerging [M]. Shanghai: Shanghai Science and Technology Education Press, 2001.

[5] Charles Domercant, Jean, Mavris, Dimitri N. Measuring the architectural complexity of military Systems-of-Systems[C]//Proceedings of IEEE Conference, 2011.

[6] Albert Network Center Warfare and Complexity Theory [M]. Electronic Industry Press, 2004: 69-79.

[7] LUO Xiao-ming, ZHU Yan-lei, HE Rong. Study on Contribution Evaluation of Weapon Equipment Combat System Based on SEM [J]. Equipment College, 2015,26 (5): 1-6.

[8] Kalloniatis A, Fairbairn D E. A new paradigm for dynamical modelling of networked $\mathrm{C} 2$ processes[C]. Proceeding of the 13th International Command and Control Research and Technology Symposium. 2008: 17-19.

[9] Domercant J C, Mavris D N. Measuring the architectural complexity of military Systems 